\title{
¿Interés público o beneficio particular? El agua en la comarca lagunera (1881-1910)
}

\author{
Guadalupe Villa Guerrero \\ INSTITUTO MORA
}

\begin{abstract}
En este ensayo se muestra cómo la folletería fue utilizada como arma política por los dos partidos en contienda sobre la distribución y el uso del agua del río Nazas.
\end{abstract}

$\mathrm{L}$ a distribución y el uso del agua del río Nazas fue raíz y razón de acres disputas entre agricultores de Durango y Coahuila, así como entre los gobiernos de ambos estados. La "cuestión del Nazas" - como se le dio en llamar- comprendía múltiples y muy complejos problemas que hicieron de ella, durante casi tres décadas, una controversia larga y reiterada. Reflejo de esta época son 33 de los 92 folletos que vieron la luz pública entre 1881 y 1909.

Puede decirse que la querella por el agua en la región lagunera tuvo como punto de partida el sonadísimo litigio entre Jesús González Treviño y Santiago Lavín. Su posición económica y so. cial, aunada a los cuantiosos intereses que ambos representaban, provocaron que el debate se llevara a extramuros de La Laguna.

Los González TreviÑo ${ }^{1}$

Al inicio de la década de los ochenta hubo en la comarca lagunera una inversión importante de capitales pro-

1 Cerutti, Burguesía, 1992. 
venientes del estado de Nuevo León, entre ellos los de las familias Madero y González Treviño, quienes, además de estar emparentados, eran socios en múltiples negocios.

Los González Treviño eran un clan importante desde el punto de vista del comercio, la industria, la minería y la tenencia de la tierra. Miembros de esta familia estaban vinculados matrimonialmente con otras muy destacadas; Mercedes González Treviño, por ejemplo, era esposa de Francisco Madero, y ambos, padres de Francisco I. Madero.

Originalmente fue a través del otorgamiento de créditos hipotecarios, para la producción y el aprovisionamiento de mercancías e implementos agrícolas, que Jesús González Treviño se convirtió en pocos años en uno de los grandes propietarios de La Laguna, llegando a ejercer un gran peso en la actividad agrícola de la región.

En 1877 González Treviño, en sociedad con otros capitalistas, abrió una sucursal refaccionaria en Villa Lerdo y concedió un primer préstamo de 45000 pesos a los herederos de Juan Ignacio Jiménez - uno de los más grandes latifundistas de Durango-, los cuales enfrentaban grandes dificultades para administrar sus bienes. ${ }^{2} \mathrm{El}$ fraccionamiento del latifundio de Juan Ignacio Jiménez en aquellos años se

${ }^{2}$ A mediados del siglo XIX, tres hacendados eran dueños absolutos de las tierras laguneras: Juan Ignacio Jiménez y Juan Nepomuceno Flores de la porción duranguense; Leonardo Zuloaga de la de Coahuila. Las guerras de Reforma e Intervención afectaron profundamente el poderío económico de aquellos propietarios que requirieron de créditos para seguir operando. debió al endeudamiento ocasionado por la repartición hereditaria, como ocurrió una década después con las propiedades de otro gran terrateniente, Juan Nepomuceno Flores.

A la muerte de Jiménez se crearon tres grandes fracciones que se repartieron entre los numerosos herederos: dos de ellas, constituidas por las tierras de Santa Rosa, Santa Cruz y dos tercios de la hacienda de Sacramento, fueron divididas en partes iguales entre los seis hijos resultantes de su primer matrimonio con María de la Luz Basoco.

La tercera fracción pasó a su segunda esposa, Eloísa San Martín, y a sus dos hijas. Las propiedades de Jiménez, como ya señalé, habian sido ampliamente hipotecadas y, tal como solía suceder en casos similares, los herederos prefirieron cederlas a los acreedores. Fue así como el endeudamiento y las hipotecas amenazaron con convertirse en una forma de acaparamiento de tierras a bajo precio.

González Treviño entró en posesión de las haciendas ya mencionadas, a las que luego sumó, de la testamentaría de Eduardo Ávila, El Alamito, la más fértil de las porciones correspondientes al latifundio de San Lorenzo de La Laguna. Al iniciar la década de los ochenta aquél se había convertido en propietario de la totalidad de la herencia de los Jiménez Basoco, que incluía la presa de Calabazas, así como una parte del Alamito.

En 1881 los hermanos González Treviño vendieron la mitad de sus pertenencias a Ramón R. Luján y Félix F. Maceyra, comerciantes de Chihuahua, en 340000 pesos. La porción que re- 
tuvieron aquéllos fue valuada en 280000 pesos. Visto así en su conjunto, el valor de las tierras de Jiménez Basoco y del Alamito era de 620000 pesos. Jesús González Treviño, junto con sus hermanos Mariano y José, reorganizaron la sociedad J. González Treviño Hermanos con el fin de administrar las haciendas de La Laguna. Con el tiempo, Luján adquirió prácticamente todas las tierras inicialmente cedidas a los González Treviño, con excepción de 7000 hectáreas pertenecientes al Alamito, sobre las cuales establecieron la hacienda El Pilar.

\section{LOS LAVÍN ${ }^{3}$}

Santiago Lavín, comerciante y prestamista originario de Aedo, provincia de Santander, España, llegó a La Laguna a mediados de la década de los sesenta como arrendatario de las tierras de Jiménez, y desde 1878 estuvo en el centro de las disputas sobre el uso de las aguas del Nazas.

En 1879, Lavín concedió a Juan Ignacio un préstamo hipotecario de 164000 pesos sobre las siguientes propiedades: hacienda de Relámpago, tercera parte de la de Sacramento y tierras situadas al oeste de Santa Rosa y Relámpago. Eloísa San Martín, segunda esposa de Juan Ignacio Jiménez y heredera del crédito en cuestión, no pudo saldar la deuda, por lo que las tierras y la presa de Santa Rosa pasaron a Lavin, quien constituyó la hacienda de Noé.

${ }^{3}$ Plana, Reino, 1991.
Sobre esas tierras fue construida en 1883 la línea del Ferrocarril Central, y en ellas se erigió la estación de Villa Lerdo, que en los años siguientes se convertiría en el núcleo urbano de Gómez Palacio.

La hacienda de Noé o Perímetro de Lavín abarcaba, en 1905, una superficie total de 47245 hectáreas, si bien la extensión cultivada tan sólo comprendía 9267 hectáreas subdivididas en catorce ranchos. Tras la muerte de Santiago, los herederos constituyeron la sociedad Santiago Lavín y Sucesores, que en 1907 tomó el nombre de Compañía Algodonera e Industrial de La Laguna, S. A.

LOS PROBLEMAS

Las aguas del río Nazas irrigaban extensos y fértiles campos pertenecientes a la municipalidad de Villa Lerdo, partido de Mapimí, en donde algunos agricultores habían realizado obras hidráulicas para hacer rendir al máximo el vital líquido. Entre éstas estaban la presa de Calabazas, propiedad de González Treviño Hermanos, y la de Santa Rosa, de Lavín y Compañía. Desde la primera -la más importante- el río descendía hasta tocar con su margen derecha terrenos correspondientes al estado de Coahuila, cuyos labradores, a su vez, lo aprovechaban aunque con menos fortuna por estar colocados en segundo término y en situación geográfica inferior. Sin embargo, era la presa de Santa Rosa la que consideraban los agricultores coahuilenses que impedía el curso natural 
del río Nazas, con el consiguiente perjuicio para ellos.

En 1881, J. González Treviño Hermanos formularon una denuncia contra Santiago Lavín ante el juez de letras de Mapimí, acusándolo de realizar una obra nueva con el objeto de prolongar la presa de Santa Rosa. Los quejosos señalaban que, luego de recibir Lavín los terrenos y la presa relacionados, comenzó a ensanchar y profundizar el canal hasta el doble de su extensión, acaparando el agua y constituyéndose en un peligro para la seguridad de los habitantes de Villa Lerdo.

El Ayuntamiento de aquella población ordenó a Lavín suspender la ejecución de la obra, sin embargo, éste optó por llevar su proyecto adelante. El problema tuvo inmediatas consecuencias administrativas y políticas. Evaristo Madero, quien había sido elegido gobernador de Coahuila en 1880 , trató de defender, a través del poder político que representaba, los intereses que el sector mercantil de Monterrey tenía en la región. Por experiencia propia conocía los distintos aspectos que ligaban la economía algodonera a la industria textil regional, y también las implicaciones económicas de la cuestión del Nazas. El 18 de febrero de 1881, Madero, en calidad de gobernador, emitió un decreto que extendía la jurisdicción de San Pedro a todas las tierras que pertenecían a Matamoros de La Laguna, situadas sobre la orilla izquierda del Nazas, punto limítrofe con el estado de Durango. Las tierras en cuestión comprendian las propiedades más directamente ligadas a los intereses de los comerciantes y fabricantes de Monterrey que habían inver- tido en la zona. Esta medida, por sí sola, no era suficiente para resolver el control de las aguas del Nazas, pero sentó precedente sobre el modo de influir en la vida económica local a través del instrumento del poder político.

El gobernador de Durango, Irancisco Gómez Palacio, sostenía que en ausencia de una legislación federal sobre las aguas superficiales, las únicas autoridades competentes para resolver las disputas entre los propietarios eran los tribunales ordinarios. Sin embargo, cuando los nuevos intereses no coincidieron más con la realidad anterior, se intentó someter los hechos a la voluntad de los grupos en conflicto, como sucedió después. Bl 16 de mayo de 1881, los agricultores de San Pedro, con el consentimiento tácito del gobernador Evaristo Maldero, se presentaron en la presa de Santa Rosa con 300 hombres armados y no sólo destruyeron las obras inicialdas por Lavín, también incendiaron la habitación del celador de las compucrtas de la presa, mantuvieron secuestrado por el breve espacio de dos horas a Ulpiano Lavín, interrumpieron la línea telegráfica y lograron interceptar un mensaje de auxilio dirigido al jefe cle la fuerza federal establecida en Matamoros de La Laguna.

El gobernador de Durango exigió al de Coahuila la reparación de perjuicios, aprehensión de los culpables, satisfacciones al estado y seguridades de que no se repetiría el agravio. José María Garza Galán, quien había sucedido en la gubernatura a Madero, respondió que la verdadera causa de lo acontecido obedecía a que había pen- 
diente una cuestión de límites que tornaba inciertas las fronteras de sus respectivos territorios y, por tanto, los consiguientes derechos para el uso de las aguas del río. Proponía que ambos gobiernos realizaran un estudio del problema territorial a fin de determinar si había existido o no invasión a Durango. En respuesta, el gobernador Francisco Gómez del Palacio envió un ocurso a la Suprema Corte de Justicia y promovió controversia contra el estado de Coahuila.

\section{LA FOLLETERÍA}

Es interesante hacer notar que Rodrigo Durán, abogado de J. González Treviño Hermanos, inició un prolongado debate con el abogado de la parte contraria utilizando la folletería como medio de expresión. Su propósito era ganarse no sólo el apoyo de la opinión pública convenciéndola de que era una causa justa para el bien común, sino llamar la atención de las autoridades hacendarias convenciéndolas de que, la falta de agua suficiente para el riego, provocaría magras o nulas cosechas $y$, con ello, pérdida de ingresos por la vía de impuestos y, finalmente, granjearse a los magistrados de la 2a. sala del Supremo Tribunal de Justicia al simplificar, mediante el folleto, la lectura del voluminoso expediente facilitándoles la toma de decisiones. Según sus propias palabras, era un asunto que

no solo atañe a los denunciantes, sino al estado en general bajo el punto de vista hacendario. Al estado no puede ser indiferente el monopolio del agua en

¿INTERÉS PÚBLICO O BENEFICIO PARTICULAR? favor de una persona, reconocido como está en los principios de economía política, que la división de los elementos de riqueza es favorable a la felicidad de los pueblos. ${ }^{4}$

La respuesta de Ladislao López Negrete, abogado de Santiago Lavín, no se hizo esperar: 5

El licenciado Rodrigo Durán ha creído conveniente dar a la prensa su informe de interdicto $[\ldots]$ motivando la publicación en que al estado no puede ser indiferente el monopolio del agua en favor de una persona, y pretendiendo captarse la opinión pública a favor de sus poderdantes. Mi parte se somete con todo gusto al fallo inapelable de tan respetado juez, y decidirá quién es el monopolizador del agua.

A mi modo de ver, los folletos publicados en Durango, Coahuila, y Monterrey - la discusión traspasó los límites de La Laguna- tienen una característica propia: ser una suerte de esgrima epistolar que busca "tocar" su objetivo. El significado y las implicaciones de haber llevado el litigio hasta el estado de Nuevo León, en un ámbito en el que los demandantes se sentían más respaldados, dejó en claro que los agricultores nunca habían cooperado entre sí para racionalizar los problemas del área. Además, las implicaciones políticas que podían originar los ribereños del lado del es-

' Informe presentado a la 2a. sala del Supremo Tribunal de Justicia, 1882.

${ }^{5}$ Informe del licenciado Ladislao López Negrete, 1882. 


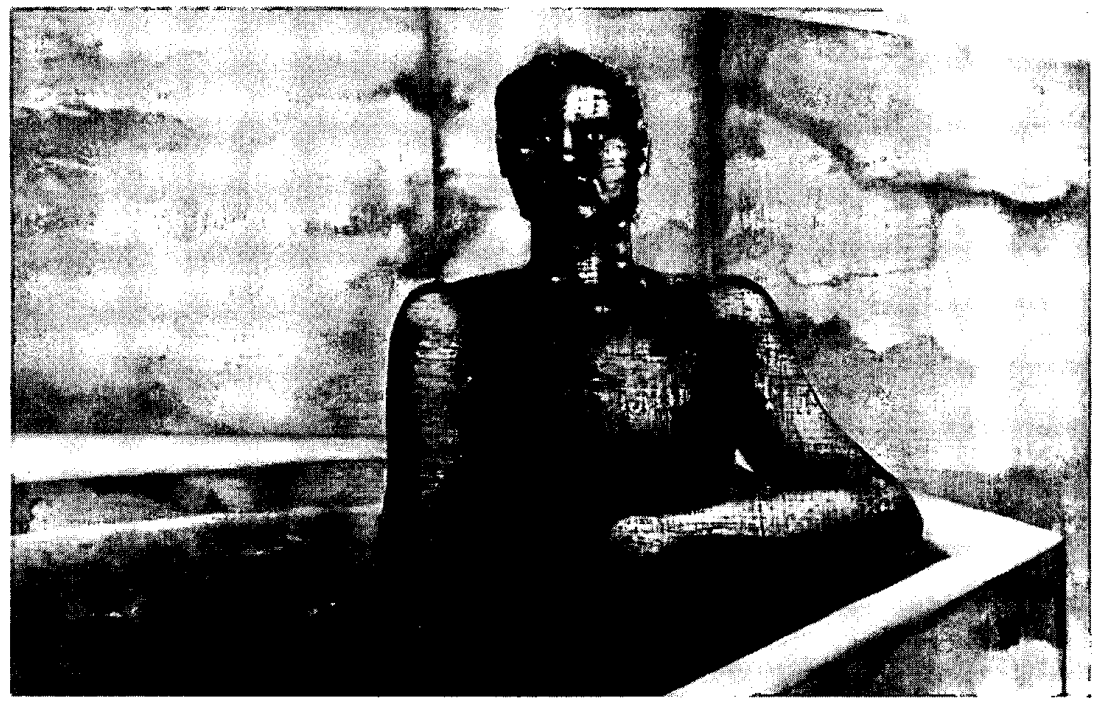

tado de Durango o los del estado de Coahuila, eran inimaginables. En cada lado se encontraban varias de las fortunas más importantes de la república acostumbradas a gozar del favor gubernamental.

El resultado de aquel litigio fue que se obligó a Lavín a suspender su obra, aunque el resto de las demandas no prosperaron: demolición de la obra, indemnización de perjuicios y condena de pago de costas personales y procesales.

\section{NUEVOS TIEMPOS-VIEJOS PROBLEMAS}

Cuando parecía que "las aguas volvían a su nivel", se sumó, en 1885 , un ele- mento más de disolución social: el establecimiento de la Compañía Agrícola del Tlahualilo Limitada, que proyectaba explotar el cultivo del algodón aprovechando para ello un gran caudal del Nazas. Desde sus inicios, la compañía atravesó por un sinfín de dificultades, ya que sus intenciones levantaron una fuerte oposición entre los agricultores de Durango y Coahuila. Alrededor de 69 terratenientes de La Laguna iniciaron una campaña ante el Ministerio de Fomento en favor de la confirmación de sus derechos preexistentes sobre las aguas del río.

Ahora bien, desde el punto de vista del gobierno central, cada vez se tornaba más difícil justificar un mayor otorgamiento de derechos sobre el agua a los poderosos intereses ubicados en 
ambos estados. Parecía que las nuevas inversiones económicas sólo conducían a cada vez más serios e incesantes conflictos. Ninguna autoridad pública había logrado convencer a los propietarios de las tierras aledañas al río de reunirse y resolver sus diferencias o sus problemas y preocupaciones comunes. En lugar de ello, una ola de inversiones había llevado a más conflictos, temores y recriminaciones entre industriales, magnates del algodón y las municipalidades. Todos necesitaban la seguridad de poder contar con un mayor volumen de agua a medida que crecían sus operaciones o las poblaciones. En La Laguna hubo una verdadera explosión de actividades: un rápido incremento en la producción algodonera, una creciente capacidad industrial, altas ganancias, una fuerte alza en el valor de la tierra y un rápido crecimiento de los pueblos, que ahora se convertían en pequeñas ciudades.

Las autoridades pronto se dieron cuenta de que la apertura de nuevas tierras al cultivo no era el único problema que debian afrontar para racionalizar el agua. Se estaba dando un incremento sostenido de las labores algodoneras y se estaban reutilizando antiguas obras de irrigación capaces de acarrear más agua que antes.

La situación de los ribereños del alto y del bajo Nazas no fue resuelta. La revolución impidió retomar la dis- cusión de una controversia que habría de dirimirse en el campo del derecho, la agricultura y la diplomacia.

\section{BIBLIOGRAFÍA}

-Cerutti, Mario, Burguesía, capitales e industria en el norte de México. Monterrey y su ámbito regional (1850-1910), Alianza Editorial/UANL, México, 1992, 383 pp.

-Informe presentado a la $2 a$. sala del Supremo Tribunal de Justicia del estado de Durango por el licenciado Rodrigo Durán como apoderado de los señores J. González Treviño hermanos en el interdicto de obra nueva promovido contra don Santiago Lavín por la prolongación de la presa de Santa Rosa al brazo dere. cho del rio Nazas, Imprenta de La Mariposa por Francisco Vera, 2a. calle de San Juan de Dios, núm. 5, Durango, 1882, 36 pp. y un croquis.

-Informe dellicenciado Ladislao López Negrete por don Santiago Lavín en el interdicto de obra nueva promovido por los señores J. González Treviño hermanos contra la prolongación de la presa de SantaRosa albrazo derecho del rio Nazas, Imprenta de la Mariposa dirigida por Francisco Vera, 2a. calle de San Juan de Dios, núm. 5, Durango, 1882,51 pp. y un croquis.

-Plana, Manuel, El reino del algodón en México. La estructura agraria de La Laguna (1855-1910), Patronato del 'Teatro Isauro Martínez/CNCA, Torreón, Coah., 1991, 275 pp. 


\section{SECUENCIA}

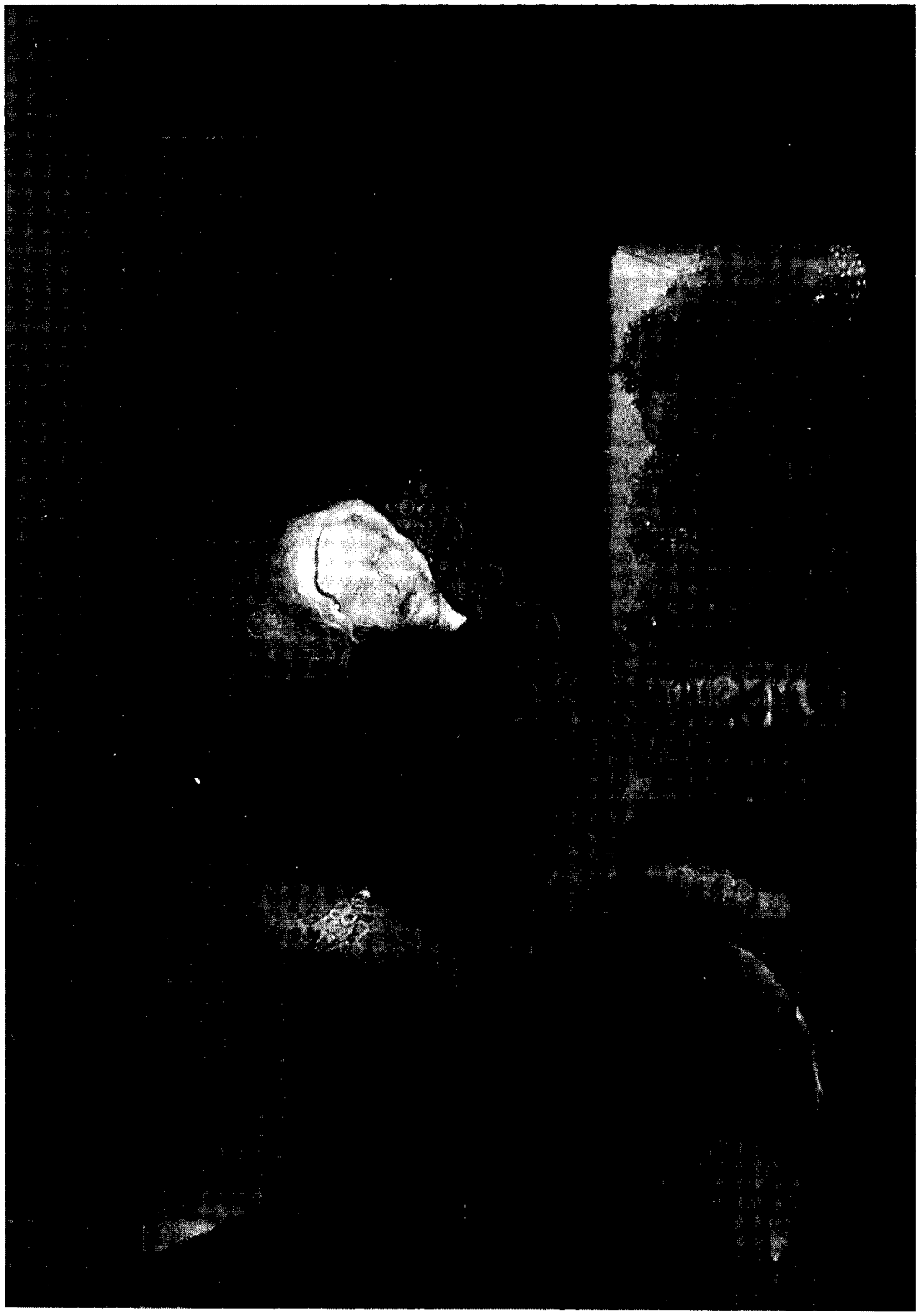

